\title{
Influencia de los patrones de uso de la tierra en la calidad de las aguas superficiales de la subcuenca del río Virilla, Costa Rica
}

\author{
Influence of Land Use Patterns in the Quality of Surface \\ Waters of the Virilla River Sub-Basin, Costa Rica
}

\author{
Jorge Herrera-Murillo ${ }^{1}$ \\ Deivis Anchía-Leitón ${ }^{2}$ \\ José Félix Rojas-Marín ${ }^{3}$ \\ Diana Mora-Campos ${ }^{4}$ \\ Alejandra Gamboa-Jiménez ${ }^{5}$ \\ María Chaves Villalobos ${ }^{6}$ \\ Universidad Nacional, Costa Rica
}

\section{Resumen}

Se analizó la relación existente entre los patrones de uso de la tierra y la calidad química de los cuerpos de agua superficial que conforman la subcuenca del Río Virilla, Costa Rica. Para ello, se seleccionaron un total de 64 sitios de monitoreo, distribuidos en las 15 microcuencas que la integran, donde se realizaron un total de 04 muestreos, en el período comprendido entre noviembre 2014 a

1 Coordinador del Laboratorio de Análisis Ambiental, Escuela de Ciencias Ambientales, Universidad Nacional, Costa Rica, jorge.herrera.murillo@una.cr

2 Investigador del Laboratorio de Análisis Ambiental, Escuela de Ciencias Ambientales, Universidad Nacional, Costa Rica, deivis.anchia.leiton@una.cr

3 Investigador del Laboratorio de Análisis Ambiental, Escuela de Ciencias Ambientales, Universidad Nacional, Costa Rica, jose.rojas.marin@una.cr

4 Directora de Calidad del Laboratorio de Análisis Ambiental, Escuela de Ciencias Ambientales, Universidad Nacional, Costa Rica diana.mora.campos@una.cr

5 Directora de Análisis Fisicoquímicos del Laboratorio de Análisis Ambiental, Escuela de Ciencias Ambientales, Universidad Nacional, Costa Rica, alejandra.gamboa@una.cr

6 Investigadora del Laboratorio de Análisis Ambiental, Escuela de Ciencias Ambientales, Universidad Nacional, Costa Rica, maria.chaves.villalobos@una.cr

Este artículo corresponde a la ponencia presentada en el I Congreso Centroamericano de Ciencias de la Tierra y el Mar, realizado en San José, Costa Rica, del 13 al 16 de noviembre de 2017. 
diciembre 2015. A las muestras recolectadas se les analizaron los siguientes parámetros: Potencial de Hidrogeno $(\mathrm{pH})$, conductividad, turbiedad, sólidos suspendidos totales (SST), sólidos sedimentables, Demanda Bioquímica de Oxigeno (DBO), Demanda Química de Oxigeno (DQO), amonio $\left(\mathrm{NH}_{4}^{+}\right)$, nitrito $\left(\mathrm{NO}_{2}^{-}\right)$, nitrato $\left(\mathrm{NO}_{3}^{-}\right)$, nitrógeno total, cloruro $\left(\mathrm{Cl}^{-}\right)$, sulfato $\left(\mathrm{SO}_{4}^{2-}\right)$, sodio, calcio, potasio, magnesio, aluminio, hierro, manganeso, cobre, cromo, níquel, zinc, plomo, fósforo total, oxígeno disuelto y temperatura. El análisis de clúster basado en los resultados de los análisis químicos en las muestras de agua superficial, evidenció que las 15 microcuencas se pueden agrupar en seis clústers, donde los grupos I-IV mostraron una mayor contribución de contaminantes derivados de la descarga de aguas residuales provenientes de las áreas urbanizadas. El estudio de correlación de Spearman reveló, que con el incremento de la urbanización, la carga contaminante de DQO, $\mathrm{DBO}, \mathrm{SST}, \mathrm{NH}_{4}^{+}$y $\mathrm{NO}_{2}^{-}$en los ríos aumentó en comparación con aquellos en donde predomina el uso agrícola de la tierra y los bosques.

Palabras clave: contaminación del agua, cuenca hidrográfica, nutrientes, análisis clúster

\begin{abstract}
We analyzed the relation between land use patterns and chemical quality of surface water bodies that make up the sub-basin of the Virilla River, Costa Rica. For this purpose, a total of 64 monitoring sites were selected, distributed in the 15 micro-basins that make up the sub-basin, where a total of four samples were drawn between November 2014 and December 2015. The following parameters were analyzed for the samples collected: Hydrogen potential $(\mathrm{pH})$, conductivity, turbidity, total suspended solids (TSS), sedimentary solids, Biochemical Oxygen Demand (BOD), Chemical Oxygen Demand (COD), ammonium (NH4+), nitrite (NO2-), nitrate (NO3-), total nitrogen, chloride (Cl-), sulfate (SO42-), sodium, calcium, potassium, magnesium, aluminum, iron, manganese, copper, chromium, nickel, zinc, lead, total phosphorus, dissolved oxygen and temperature. Cluster analysis, based on the results of chemical analyses of surface water samples, showed that the 15 micro watersheds could be grouped into six clusters, where groups I-IV showed a greater contribution of pollutants derived from the discharge of wastewater from urbanized areas. The Spearman correlation study revealed that, with increasing urbanization, the pollutant load of COD, BOD, TSS, NH4+, and NO2- in rivers increased, compared to those where agricultural land and forest use predominate.
\end{abstract}

Keywords: water pollution, basin, nutrients, cluster analysis.

\title{
Introducción
}

Las condiciones hidrológicas de una cuenca dependen de la interrelación entre factores geológicos, geomorfológicos, así como de los patrones de uso de la tierra predominantes. De esta forma, la calidad de los cuerpos de agua que integran la cuenca se ven afectados por factores, tanto de origen natural como antropogénico (Songyan et al., 2016). Para entender la compleja relación entre los patrones de uso del suelo y la calidad del agua se deben integrar dos dimensiones de análisis, primero, los efectos de las categorías de uso del suelo en las variables de calidad del agua, y posteriormente, las escalas espaciales en las que existen los vínculos más 
fuertes (Uriarte et al., 2011). En este análisis se debe tomar en cuenta la variación espacial de las actividades antropogénicas que son responsables de la generación y transporte de contaminantes terrestres, tanto a partir de fuentes puntuales de descarga que son fáciles de evaluar como por la contaminación difusa, que implica la escorrentía de contaminantes a los cuerpos de agua superficial. Las actividades desarrolladas por el hombre alteran los procesos ya existentes, de manera que, incorporan nuevas fuentes de contaminación a los cuerpos de agua superficial, lo que provoca que el carbono, los nutrientes y otros contaminantes, como el cloruro y el calcio registren un comportamiento más dinámico (Kaushal y Belt, 2012).

Los cambios en los patrones de uso de la tierra, incluyendo la deforestación, la fragmentación de hábitats continuos, la canalización y cambios en el cauce de los ríos han dado como resultado la erosión del suelo, la pérdida de biodiversidad acuática, la disminución de caudales y la degradación de la calidad del agua (Walling y Fang 2003, Tong et al. (2009), Miserendino et al. (2011)). Adicionalmente, el crecimiento de las áreas urbanas y con ello las presiones asociadas al desarrollo y la falta de planificación del uso del suelo, contribuyen continuamente a la degradación de los recursos hídricos (Saroj et al., 2013), lo que se traduce en trastornos ambientales que modifican los ecosistemas acuáticos, manifestándose sobre la productividad de sistemas naturales donde se da interacción humana (Carbone et al., 2013).

El desarrollo urbano causa modificaciones significativas en el tiempo y el volumen de las escorrentías (Wear et al., 1998). Cuando una cuenca hidrográfica se encuentra cubierta, en un alto porcentaje, por infraestructuras como edificaciones, concretos y pavimentos se produce una mayor escorrentía superficial (Quesada, 2012), lo que propicia el aporte en menor tiempo y mayor cantidad de contaminantes producidos. Por su parte, las áreas agrícolas y ganaderas que ocupan gran parte del paisaje en las cuencas, son fuentes importantes de contaminación y en general representan efectos que incluyen aumento en la carga de sedimentos, las concentraciones de sales, metales (sodio, patasio, calcio, magnesio, manganeso, hierro, etc), productos agroquímicos y agentes patógenos, así como cambios en el régimen térmico (Cisneros, 2005). Esta capacidad se ve acelerada debido a las precipitaciones que transportan el suelo erosionado con restos de plaguicidas, principalmente en zonas de fuerte pendiente. 
En Costa Rica se han generado diversos instrumentos de gobernanza que no garantizan una verdadera sustentabilidad del recurso hídrico, ya que adolecen de la incorporación de la dimensión del ordenamiento territorial donde se establezca claramente las regiones de producción y de recarga hídrica, y que permita, a partir de estas premisas, planificar el desarrollo agropecuario, industrial, inmobiliario y turístico. Estas carencias en materia de planificación aunadas a la falta de un enfoque integrado de gestión de cuencas, causan efectos ambientales que repercuten en la potencialidad hídrica del país.

Los cambios en las características de calidad del agua dentro de las cuencas, han generado preocupación e interés por llevar a cabo estudios que permitan conocer cuáles son los factores que motivan dichos cambios y que tan significativo es el aporte de cada uno de ellos (Sangani et al., 2015). El presente estudio analizó la influencia de los diferentes tipos de uso del suelo asociados con la actividad antrópica, sobre las características químicas de los cuerpos de agua superficial y por ende, su calidad en las 15 microcuencas que integran la subcuenca del Río Virilla (Tabla 1). Este se llevó a cabo con el fin de generar un insumo que permita orientar el proceso de toma de decisiones públicas en materia de gestión integrada de cuencas hidrográficas en el país.

Tabla 1. Porcentajes de área por tipo de uso de la tierra para cada microcuenca. 2015

\begin{tabular}{|l|c|c|c|c|c|c|c|c|}
\hline \multirow{2}{*}{ Microcuenca } & & \multicolumn{6}{|c|}{ Porcentaje de área por tipo de uso de la tierra } \\
\cline { 2 - 9 } & Código & Agrícola & Urbana & Bosque & $\begin{array}{c}\text { Charral } \\
\text { y Tacotal }\end{array}$ & Pastos & $\begin{array}{c}\text { Terreno } \\
\text { descubierto }\end{array}$ & $\begin{array}{c}\text { Sin } \\
\text { identificar }\end{array}$ \\
\hline Río Bermúdez & BE & 33 & 43 & 14 & 3 & 7 & 0 & 0 \\
\hline Río Segundo & RS & 31 & 19 & 30 & 2 & 16 & 0 & 2 \\
\hline Río Tibás & TIB & 34 & 7 & 21 & 4 & 33 & 0 & 1 \\
\hline Río Ciruelas & CI & 23 & 23 & 21 & 2 & 24 & 0 & 7 \\
\hline Río Uruca & UR & 12 & 5 & 59 & 4 & 18 & 1 & 0 \\
\hline Río Pará & PAR & 1 & 4 & 31 & 2 & 60 & 0 & 2 \\
\hline Río Macho & MA & 0 & 4 & 14 & 3 & 72 & 0 & 7 \\
\hline
\end{tabular}


Jorge Herrera-Murillo - Deivis Anchía-Leitón - José Félix Rojas-Marín

Diana Mora-Campos - Alejandra Gamboa-Jiménez - María Chaves Villalobos.

Influencia de los patrones de uso de la tierra en la calidad de las aguas superficiales de la subcuenca del río Virilla, Costa Rica

\begin{tabular}{|l|c|c|c|c|c|c|c|c|}
\hline \multirow{2}{*}{ Microcuenca } & & \multicolumn{6}{|c|}{ Porcentaje de área por tipo de uso de la tierra } \\
\cline { 2 - 9 } & Código & Agrícola & Urbana & Bosque & $\begin{array}{c}\text { Charral } \\
\text { y Tacotal }\end{array}$ & Pastos & $\begin{array}{c}\text { Terreno } \\
\text { descubierto }\end{array}$ & $\begin{array}{c}\text { Sin } \\
\text { identificar }\end{array}$ \\
\hline $\begin{array}{l}\text { Quebrada San } \\
\text { Francisco }\end{array}$ & SF & 16 & 71 & 12 & 1 & 0 & 0 & 0 \\
\hline Río Ipí98*/ & IP & 2 & 87 & 4 & 2 & 5 & 0 & 0 \\
\hline Río Durazno & DU & 0 & 3 & 36 & 4 & 54 & 0 & 2 \\
\hline Río Virilla & VI & 15 & 26 & 28 & 5 & 18 & 0 & 9 \\
\hline Río Jaris & JA & 1 & 0 & 79 & 0 & 20 & 0 & 0 \\
\hline Río Picagres & PI & 11 & 4 & 52 & 5 & 23 & 3 & 2 \\
\hline Río Pacacua & PA & 9 & 6 & 50 & 4 & 30 & 0 & 0 \\
\hline Río Torres & TO & 12 & 38 & 31 & 6 & 12 & 0 & 1 \\
\hline
\end{tabular}

Fuente: Elaboración Propia

\section{Materiales y métodos}

\section{Descripción del área de estudio}

La sub-cuenca del Río Virilla es un contribuyente importante de la cuenca del río Grande de Tárcoles en la vertiente del Pacífico. Cuenta con una extensión territorial de $917.6 \mathrm{~km}^{2}$, aproximadamente, y se encuentra sobre todo al norte de la provincia de San José $\left(520.4 \mathrm{~km}^{2}\right)$ y sur de la provincia de Heredia $\left(235.1 \mathrm{~km}^{2}\right)$, con pequeñas áreas en las provincias de Cartago $\left(72.1 \mathrm{~km}^{2}\right)$ y Alajuela $\left(89.1 \mathrm{~km}^{2}\right)$. En esta subcuenca habitan 2063935 habitantes, aproximadamente, lo que representa el $48 \%$ de la población del país (INEC, 2017).

El río Virilla se origina al norte del Gran Área Metropolitana de Costa Rica (GAM), en las cercanías de la Cordillera Volcánica Central. El mismo nace a $2380 \mathrm{msnm}$ y su parte más baja se encuentra a $280 \mathrm{msnm}$. Es la zona más desarrollada del país, lo que significa que tiene una gran cantidad de industria y un alto porcentaje de uso urbano. Además, este río provee numerosos servicios ecosistémicos, incluyendo una fuente de agua para uso doméstico, industrial y agrícola, desempeñando así, un papel importante para el desarrollo de la economía y la sociedad (Figura 1). 
Figura 1. Ubicación geográfica de la subcuenca del río Virilla, Costa Rica

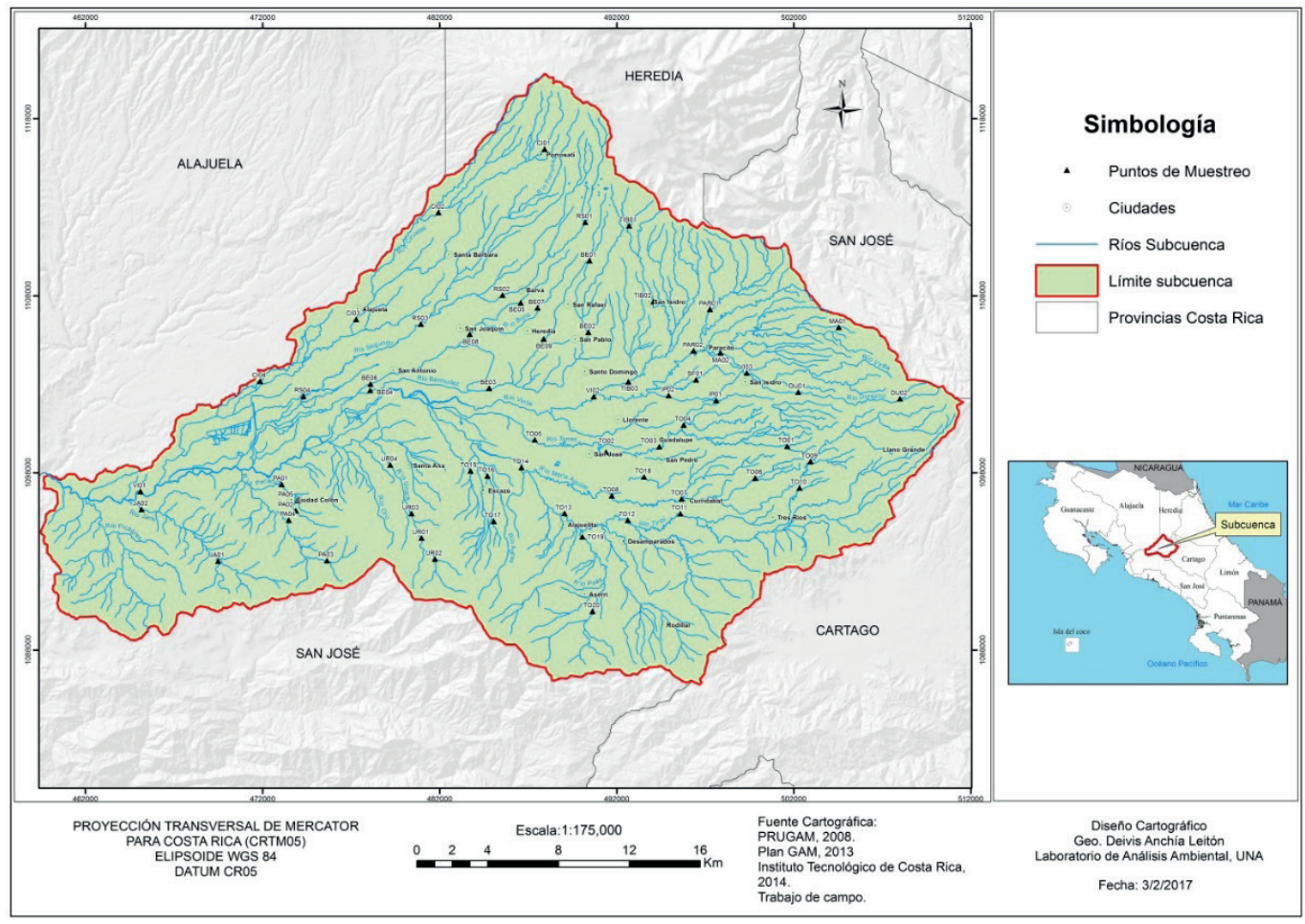

El régimen de precipitación de la subcuenca es de tipo Pácifico, con un periodo seco y lluvioso bien definidos. La época seca se registra normalmente entre mediados de noviembre a, inclusive, el mes de abril y la época lluviosa inicia en mayo y concluye a mediados de noviembre. La precipitación media anual va desde los 1500 a los $4000 \mathrm{~mm}$, marcándose una media anual de hasta los $4000 \mathrm{~mm}$ y $2000 \mathrm{~mm}$, en las partes altas y bajas, respectivamente. Los meses de setiembre y octubre suelen ser los más lluviosos, aportando aproximadamente de 17 a $18 \%$, respectivamente, de la precipitación promedio anual (IMN, 2017). Con relación al tipo de suelo existente en el área de estudio, domina el orden Andisol con 31.5 $\%$ del área total de la subcuenca. Le siguen Urbano (30.7\%), Ultisoles/ Inceptisoles (16.9\%), Ultisoles (12.8\%), Inceptisoles (11.7\%), Entisoles (1.8\%) y Vertisoles (0.4\%) (CIA, 2017) (Figura 2). 
Jorge Herrera-Murillo - Deivis Anchía-Leitón - José Félix Rojas-Marín

Diana Mora-Campos - Alejandra Gamboa-Jiménez - María Chaves Villalobos.

Influencia de los patrones de uso de la tierra en la calidad de las aguas superficiales de la subcuenca del río Virilla, Costa Rica

Figura 2. Distribución del orden de los suelos en la subcuenca del río Virilla, Costa Rica

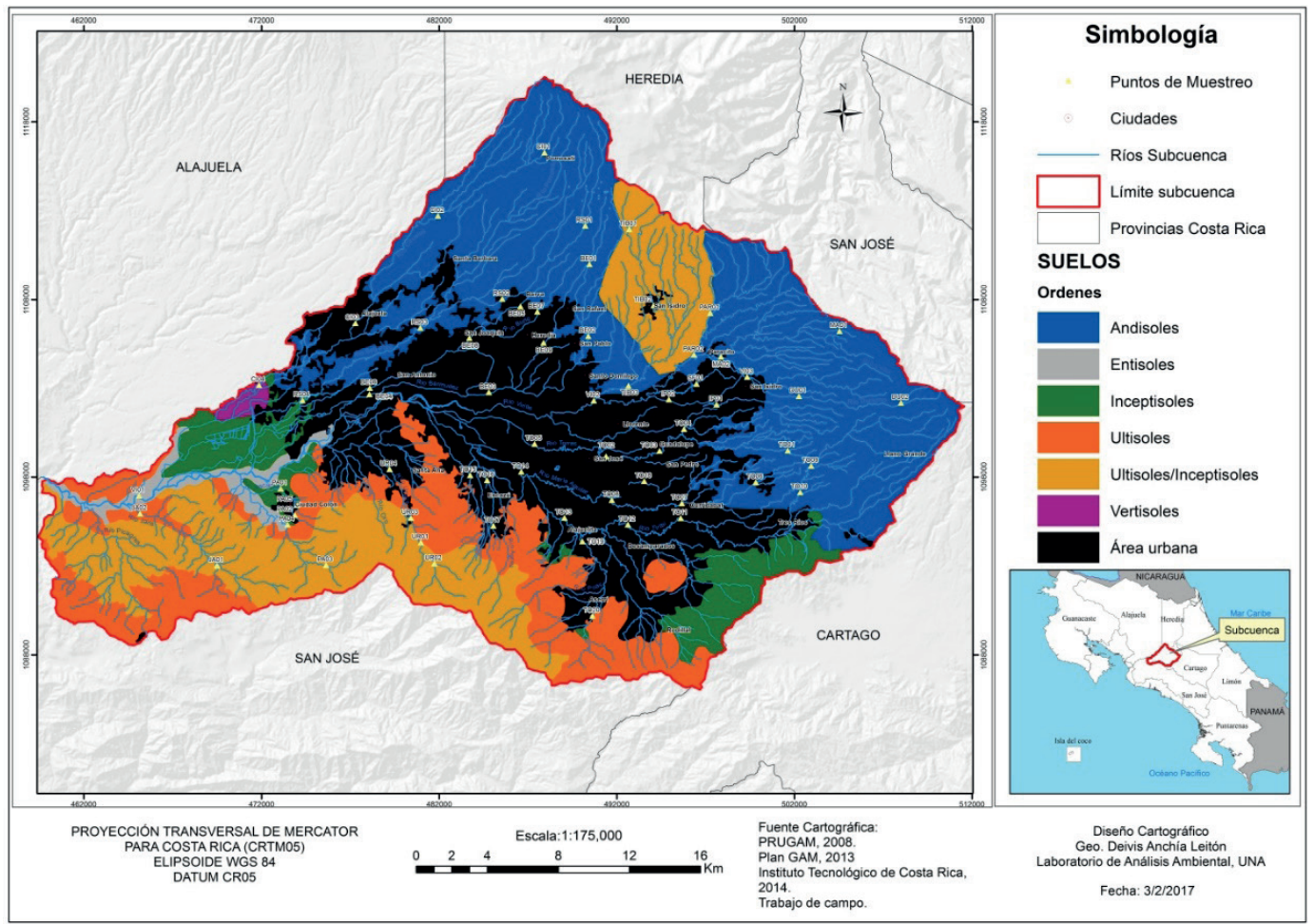

El uso del suelo fue creado por el Plan Regional de Ordenamiento Territorial de la Gran Área Metropolitana en el año 2013, donde se indica que dentro de la subcuenca se presentan ocho categorías, principalmente: áreas urbanas con un $27.6 \%$, bosques $25.9 \%$, tierras agrícolas $14.8 \%$, áreas donde no existen datos $7.8 \%$, charrales y tacotales $5.5 \%$, cuerpos de agua $0.7 \%$, pastos $18.1 \%$ y terrenos descubiertos representan $0.3 \%$ del área total de la cuenca (Figura 3). 
Figura 3. Patrones de uso de la tierra en la subcuenca del río Virilla, Costa Rica 2015

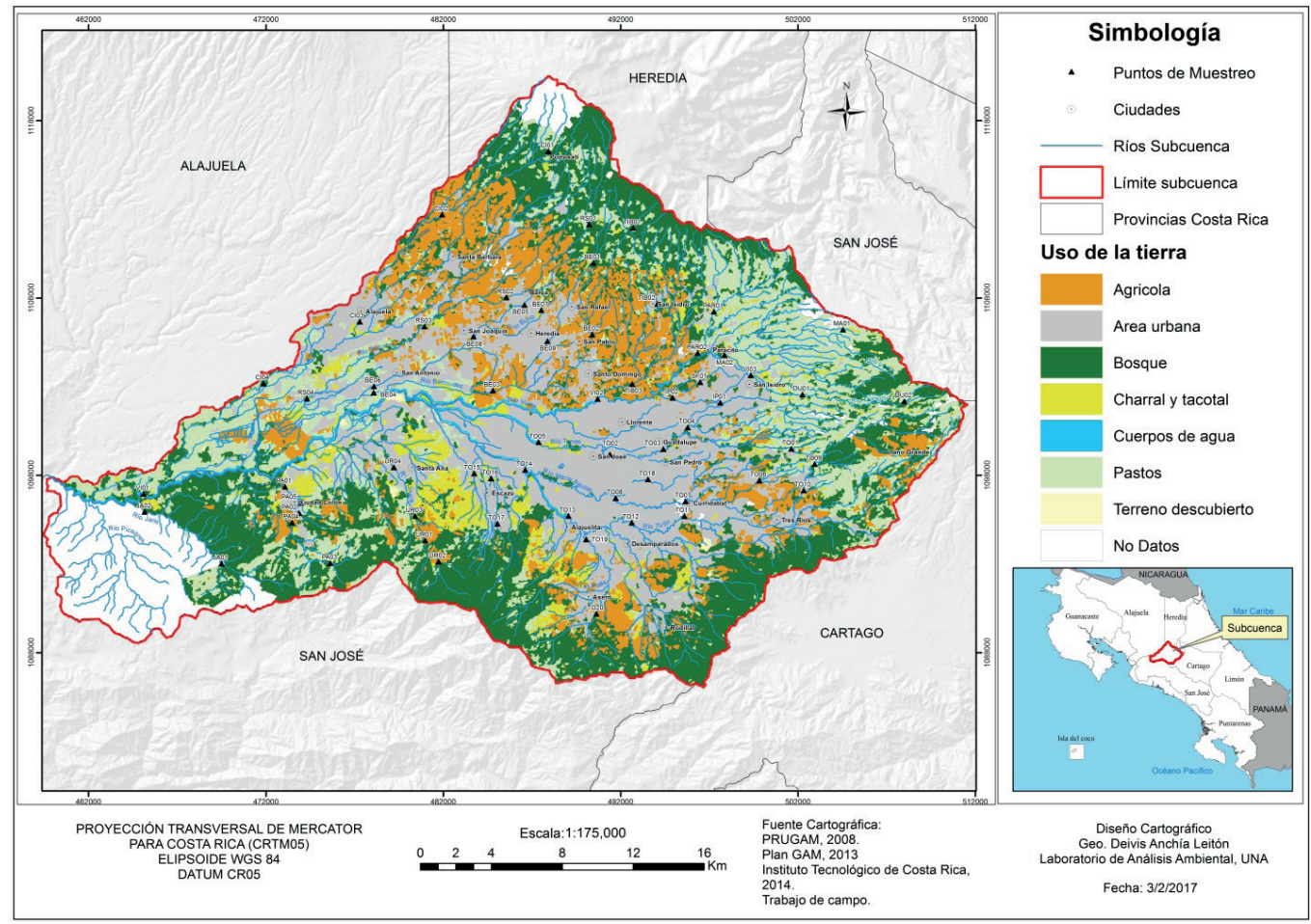

\section{Pendientes}

Utilizando el software ArcGis se generó el mapa de pendientes mediante el uso de las curvas de nivel 1:50 000 con proyección CRTM05, las cuales fueron obtenidas del Atlas Cantonal elaborado por el Instituto Tecnológico de Costa Rica. A partir de estas, se creó un modelo digital de elevación (MDE) $10 \times 10 \mathrm{~m}^{2}$ para delinear el área de la subcuenca, obtener la red de drenaje y así obtener, tanto las características topográficas como las pendientes en el área de estudio y la pendiente media (Figura 4). 
Jorge Herrera-Murillo - Deivis Anchía-Leitón - José Félix Rojas-Marín

Diana Mora-Campos - Alejandra Gamboa-Jiménez - María Chaves Villalobos.

Influencia de los patrones de uso de la tierra en la calidad de las aguas superficiales de la subcuenca del río Virilla, Costa Rica

Figura 4. Nivel de pendientes en la subcuenca del río Virilla, Costa Rica

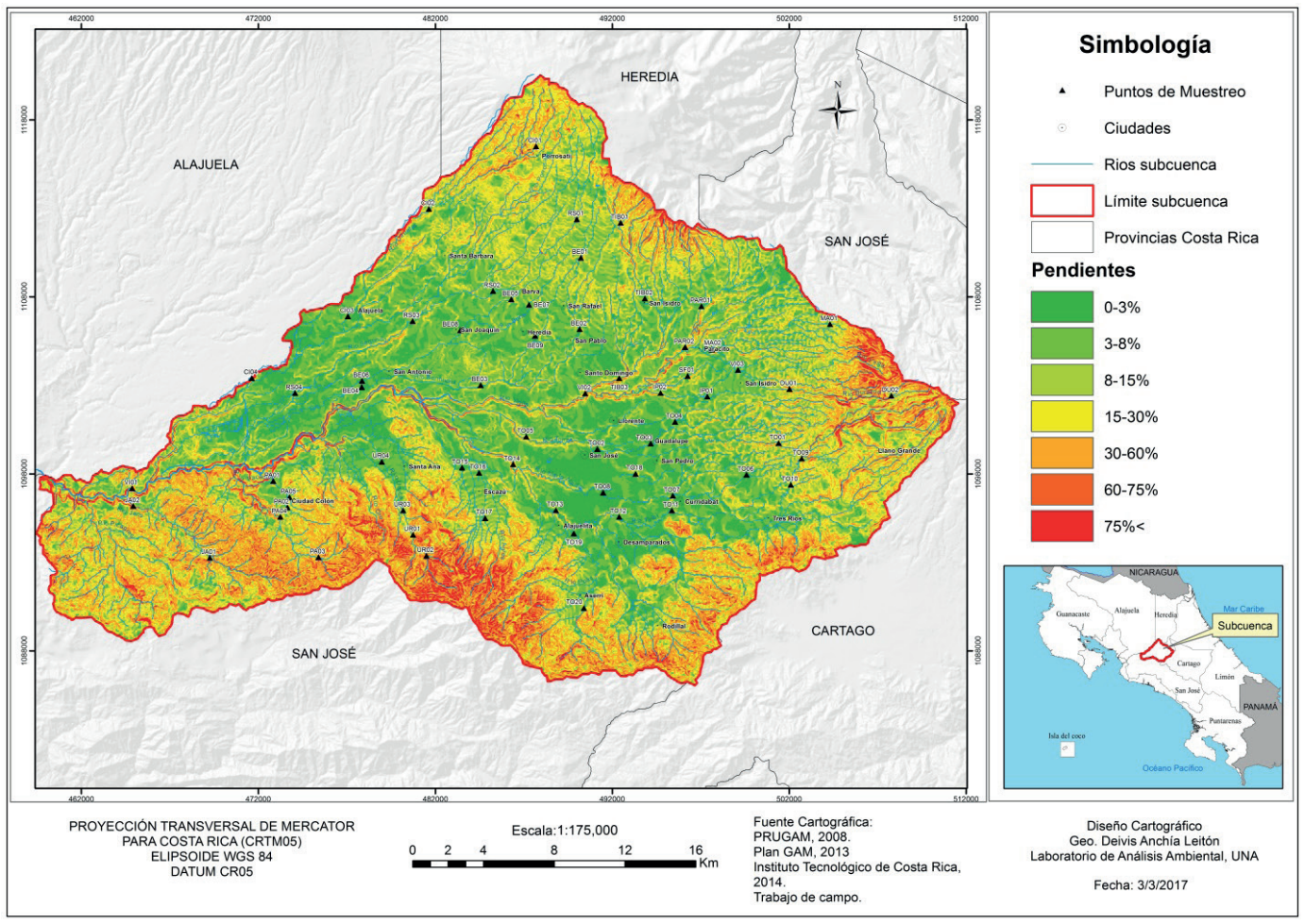

Del MDE se extrajeron siete categorías de pendiente y su porcentaje de representatividad en el área de la subcuenca:

-Categoría I (0-3\%): $13.9 \%$

-Categoría II (3-8 \%): $21.8 \%$

-Categoría III (8-15\%): $16.5 \%$

-Categoría IV (15-30\%): $20.0 \%$

-Categoría V (30-60\%): $21.9 \%$

-Categoría VI (60-75\%): $4.2 \%$

-Categoría VII (> $75 \%): 2.3 \%$

Según indica el Ministerio de Agricultura y Ganadería de Costa Rica, dependiendo del porcentaje de pendiente, las áreas pueden ser clasificadas de la siguiente forma (Tabla 2). 
Tabla 2. Clasificación de áreas según su pendiente, 2016.

\begin{tabular}{|c|c|}
\hline Pendiente & Superficie \\
\hline $0-3 \%$ & plana o casi plana \\
\hline $3-8 \%$ & ligeramente ondulada \\
\hline $8-15 \%$ & moderadamente ondulada \\
\hline $15-30 \%$ & ondulada \\
\hline $30-60 \%$ & fuertemente ondulada \\
\hline $60-75 \%$ & escarpada \\
\hline $75 \%<$ & fuertemente escarpada \\
\hline
\end{tabular}

Fuente: Elaboración propia según datos del (Ministerio de Agricultura y Ganadería, 2016).

Sitios de muestreo y calidad del agua

Se seleccionaron un total de 64 sitios de monitoreo distribuidos en las 15 microcuencas que integran la subcuenca del río Virilla, a saber: río Ciruelas (CI), río Segundo (RS), río Bermúdez (BE), río Tibás (TIB), río Para (PAR), río Macho (MA), río Durazno (DU), Quebrada San Francisco (SF), río Ipís (IP), río Torres (TO), río Rivera (RIV), río Uruca (UR), río Jaris (JA), río Picagres (PI) y río Pacacua (PA). La ubicación de los sitios respondió a criterios, como cambios importantes en el nivel de pendientes, uso del suelo, entrada y salida a importantes centros urbanos. Se realizaron un total de 4 periodos de muestreo distribuidos entre noviembre 2014 a diciembre 2015. En la toma de muestras para el análisis físico-químico por cada sitio se usaron botellas de polietileno de alta densidad de $3.78 \mathrm{~L}$ para recolectar muestras compuestas. Durante cada muestreo se recolectaron 2 submuestras de 1.89 L cada una en diferentes horas del día (mañana y tarde), y posteriormente se conservaron a $4^{\circ} \mathrm{C}$ en hieleras para transportarlas al laboratorio. Las muestras se tomaron de 0.5 a $1.0 \mathrm{~m}$ de distancia de la orilla del río y a una profundidad promedio de $20 \mathrm{~cm}$, donde no existiera turbulencia y descargas próximas de aguas residuales.

Para el análisis de metales pesados se utilizaron botellas de polietileno de baja densidad de $100 \mathrm{~mL}$, las cuales se colocaron al menos $24 \mathrm{~h}$ en un baño de ácido nítrico al $10 \%$ y se enjuagaron varias veces con agua 
desionizada antes del muestreo. Durante la ejecución del muestreo se exponía un frasco con $100 \mathrm{~mL}$ de agua MiliQ al ambiente (primera muestra del día), cumpliendo una función de blanco. Seguidamente, utilizando otro frasco recolector se realizaban tres enjuagues con la misma agua a muestrear y por último se tomaba la muestra directamente del cauce del río, conservándola a $4{ }^{\circ} \mathrm{C}$ en la hielera, para su posterior traslado al laboratorio. Las muestras de metales pesados se colectaron durante la primera toma de muestra compuesta (submuestra) en cada sitio y para cada campaña. Cada una de las muestras se identificó adecuadamente con una etiqueta que contenía un número asignado en el correspondiente plan de muestreo.

Para la medición de oxígeno disuelto $(\mathrm{mg} / \mathrm{L})$ y temperatura del agua $\left({ }^{\circ} \mathrm{C}\right)$ se usó el equipo Multiparámetro HQ40d en cada toma de submuestra. La determinación de los caudales se efectuó para los 64 sitios monitoreados en los cuatro periodos usando el método de área transversal y velocidad promedio con la ayuda de un Caudalímetro marca FLOWATCH.

Los análisis químicos realizados a las muestras recolectadas se indican en la Tabla 3. El análisis de metales trazas se efectúo en muestras sin filtrar (totales) y en muestras filtradas con filtros de nitrato de celulosa de 0,45 micras (disueltos). Antes de las mediciones, las muestras de agua fueron digeridas por acidificación con $\mathrm{HNO}_{3}(2,5 \mathrm{~mL}$ de ácido concentrado a $25 \mathrm{~mL}$ de la muestra).

Tabla 3. Métodos analíticos empleados en la evaluación de las muestras de agua recolectadas

\begin{tabular}{|l|c|c|c|}
\hline \multicolumn{1}{|c|}{ Variable } & Método & $\begin{array}{c}\text { Límite de } \\
\text { detección }\end{array}$ & Unidades \\
\hline Demanda química de oxígeno & Método estándar 5220 D & 6 & $\mathrm{mg} \mathrm{O}_{2} / 1$ \\
\hline Demanda bioquímica de oxígeno & Método estándar 5220 D & 3 & $\mathrm{mg} \mathrm{O}_{2} / 1$ \\
\hline $\mathrm{pH}$ & Método estándar 4500-H+ B & $\mathrm{NA}$ & Unidades $\mathrm{pH}$ \\
\hline Conductividad & Método estándar 2510 B & $\mathrm{NA}$ & $\mu \mathrm{S} / \mathrm{cm}$ \\
\hline Oxígeno disuelto & Método estándar 4500-O & 0.10 & $\mathrm{mg} \mathrm{O} / 1$ \\
\hline Sólidos sedimentables & Método estándar 2540 & 0.1 & $\mathrm{~mL} / 1$ \\
\hline Sólidos suspendidos totales & Método estándar 2540 D & 0.6 & $\mathrm{mg} / 1$ \\
\hline Turbiedad & Nefelométrico & $\mathrm{Na}$ & $\mathrm{NTU}$ \\
\hline Fósforo total & Método estándar 4500-P C & 0.7 & $\mathrm{mg} / 1$ \\
\hline Nitrógeno Total & Método estándar 4500-N C & 2 & $\mathrm{mg} / 1$ \\
\hline
\end{tabular}


Jorge Herrera-Murillo - Deivis Anchia-Leitón - José Félix Rojas-Marín Diana Mora-Campos - Alejandra Gamboa-Jiménez - María Chaves Villalobos.

Influence of Land Use Patterns in the Quality of Surface Waters of the Virilla River Sub-Basin, Costa Rica

\begin{tabular}{|l|c|c|c|}
\hline \multicolumn{1}{|c|}{ Variable } & Método & $\begin{array}{c}\text { Límite de } \\
\text { detección }\end{array}$ & Unidades \\
\hline Nitrito & Método estándar 4500-NO2- & 8 & $\mu \mathrm{g} / \mathrm{l}$ \\
\hline Amonio & Método estándar 4500-NH3 & 14 & $\mu \mathrm{g} / \mathrm{l}$ \\
\hline Cloruro & Método estándar 4110 & 0.10 & $\mathrm{mg} / \mathrm{l}$ \\
\hline $\mathrm{Sulfato}$ & Método estándar 4110 & 0.14 & $\mathrm{mg} / \mathrm{l}$ \\
\hline $\mathrm{Nitrato}$ & Método estándar 4110 & 0.05 & $\mathrm{mg} / 1$ \\
\hline $\mathrm{Na}$ & Método estándar 3111 & 0.11 & $\mathrm{mg} / 1$ \\
\hline $\mathrm{K}$ & Método estándar 3111 & 0.07 & $\mathrm{mg} / 1$ \\
\hline $\mathrm{Ca}$ & Método estándar 3111 & 0.8 & $\mathrm{mg} / 1$ \\
\hline $\mathrm{Mg}$ & Método estándar 3111 & 0.01 & $\mathrm{mg} / 1$ \\
\hline $\mathrm{Zn}$ & Método estándar 3111 & 0.02 & $\mathrm{mg} / \mathrm{l}$ \\
\hline $\mathrm{Al}$ & Método estándar 3111 & 1.1 & $\mu \mathrm{g} / \mathrm{l}$ \\
\hline $\mathrm{Fe}$ & Método estándar 3111 & 4 & $\mu \mathrm{g} / \mathrm{l}$ \\
\hline $\mathrm{Cr}$ & Método estándar 3111 & 3 & $\mu \mathrm{g} / \mathrm{l}$ \\
\hline $\mathrm{Cu}$ & Método estándar 3111 & 1.5 & $\mu \mathrm{g} / \mathrm{l}$ \\
\hline $\mathrm{Ni}$ & Método estándar 3111 & 2.4 & $\mu \mathrm{g} / \mathrm{l}$ \\
\hline $\mathrm{Pb}$ & Método estándar 3111 & 1.8 & $\mu \mathrm{g} / \mathrm{l}$ \\
\hline $\mathrm{Mn}$ & Método estándar 3111 & 2 & $\mu \mathrm{g} / \mathrm{l}$ \\
\hline
\end{tabular}

Fuente: Elaboración propia

\section{Análisis de los datos}

A partir de los datos obtenidos, se utilizó la prueba de KolmogorovSmirnov para determinar la normalidad de los parámetros individuales de calidad del agua, así como la prueba de Kruskal-Wallis para determinar si había diferencias significativas en la calidad del agua entre microcuencas y en las diferentes épocas del año, a un nivel de significancia de 0.05 . Para la interpretación de los resultados, se realizó un análisis jerárquico de conglomerados para agrupar las microcuencas en clases, según el uso del suelo y la química del agua. El análisis de conglomerados se corrió en conjuntos de datos normalizados por medio del método Ward y utilizando distancias euclidianas como medida de similitud. Las relaciones existentes entre los patrones de usos del suelo y los parámetros de calidad del agua fueron evaluadas a partir de la elaboración de una matriz de correlación de Spearman y un análisis de factor. 


\section{Resultados y discusión}

Los resultados promedio obtenidos para los parámetros fisicoquímicos evaluados durante las campañas de muestreo, en cada una de las microcuencas que integran la subcuenca del río Virilla, se muestran en las Tablas 4 y 5 . La normalidad de los datos de la calidad del agua se evaluó mediante la aplicación de la prueba de Kolmogorov-Smirnov (K-S), resultando que todos los parámetros, con excepción del oxígeno disuelto, presentaron una distribución no normal. Tal como se puede apreciar las microcuencas ubicadas en el sector noroeste de la subcuenca del río Virilla (RS, BE, CI y TIB) presentan concentraciones de Ca y Mg menores a las registradas para aquellas localizadas en el sureste (PI, JA, PA y UR). Esta diferencia se podría explicar debido al hecho de que el primer grupo se encuentra asentado en suelos de tipo andisol a diferencia del segundo donde predominan los suelos ultisoles, los cuales poseen condiciones de agregamiento que favorecen la lixiviación de nutrimentos, especialmente las bases $\mathrm{Ca}, \mathrm{Mg}$ y K, presentando problemas de acidez. De igual forma, los valores de $\mathrm{pH}$ entre ambos grupos de microcuencas resultaron ser significativamente diferentes $(\mathrm{p}<0.01)$.

Para determinar el nivel de contaminación de cada microcuenca debido a la presencia de metales se utilizó el índice de metal (IM), el cual entre mayor sea la concentración de un metal en comparación con su valor máximo permisible, peor es la calidad del agua. Un valor de MI $>1$ es un umbral de advertencia. Según (Tamasi y Cini, 2004), el IM se calcula utilizando la siguiente fórmula:

Donde: $\quad \mathrm{IM}=\sum_{i=1}^{n} \frac{C i}{(V M P)}$

$\mathrm{Ci}=$ concentración de cada metal

$\mathrm{VMP}=$ valor máximo permitido para cada metal 
Jorge Herrera-Murillo - Deivis Anchia-Leitón - José Félix Rojas-Marín Diana Mora-Campos - Alejandra Gamboa-Jiménez - María Chaves Villalobos. Influence of Land Use Patterns in the Quality of Surface Waters of the Virilla River Sub-Basin, Costa Rica

\begin{tabular}{|c|c|c|c|c|c|c|c|c|c|c|c|c|c|c|c|c|}
\hline & 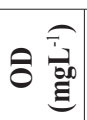 & $\begin{array}{l}\dot{J} \\
\dot{\nabla}\end{array}$ & $\begin{array}{l}\infty \\
0 \\
\sim\end{array}$ & $\underset{\sim}{\stackrel{\gamma}{r}}$ & $\stackrel{?}{\stackrel{P}{r}}$ & 守. & ñ & $\stackrel{n}{n}$ & $\begin{array}{l}\infty \\
\infty \\
\forall\end{array}$ & $\underset{r}{8}$ & $\stackrel{\Re}{\stackrel{9}{r}}$ & $\frac{m}{\infty}$ & $\stackrel{N}{\sim}$ & $\underset{\infty}{\infty}$ & $\stackrel{n}{n}$ & $\hat{n}$ \\
\hline & $\underset{0}{0}$ & $\stackrel{n}{\sim}$ & $\stackrel{\circ}{\circ}$ & $\stackrel{\partial}{\infty}$ & $\begin{array}{l}\hat{\infty} \\
-\end{array}$ & $\begin{array}{l}\stackrel{0}{0} \\
\stackrel{\sim}{ }\end{array}$ & $\stackrel{\nabla}{\partial}$ & $\begin{array}{l}\infty \\
\infty \\
\infty\end{array}$ & $\begin{array}{l}\stackrel{0}{\circ} \\
\stackrel{\sim}{0}\end{array}$ & $\stackrel{\sim}{\sim}$ & $\begin{array}{l}\infty \\
\infty \\
\infty\end{array}$ & $\hat{\tilde{v}}$ & $\stackrel{?}{\sim}$ & $\begin{array}{l}\infty \\
\dot{n} \\
\sim\end{array}$ & 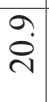 & $\vec{\sim}$ \\
\hline & 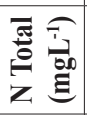 & $\stackrel{m}{\dddot{m}}$ & $\begin{array}{l}\text { ナ. } \\
\dot{J}\end{array}$ & 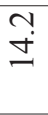 & $\hat{a}$ & $\ddot{n}$ & ন্. & $\tilde{\sigma}$ & $\hat{\curvearrowright}$ & $\stackrel{\circ}{\underset{J}{J}}$ & $\stackrel{\circ}{\circ}$ & $\Xi$ & 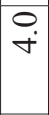 & ñ & $\mathfrak{r}$ & $\stackrel{9}{=}$ \\
\hline & 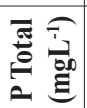 & ִָ & $\stackrel{N}{N}$ & $\begin{array}{l}\stackrel{0}{0} \\
\text { N }\end{array}$ & $\begin{array}{l}\dot{0} \\
\dot{0} \\
m\end{array}$ & $\dot{\infty}$ & $\frac{n}{\sim}$ & $\begin{array}{l}\nabla \\
\ddot{v}\end{array}$ & ন & $\begin{array}{l}\text { லे } \\
\text { ते }\end{array}$ & $\begin{array}{l}0 \\
\dot{\sim}\end{array}$ & $\frac{a}{\sim}$ & $\begin{array}{l}0 \\
\dot{n}\end{array}$ & 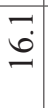 & $\hat{0}$ & $\dot{\rho}$ \\
\hline$\frac{n}{2}$ & 唔 & $\begin{array}{l}\text { ָे } \\
\stackrel{n}{n}\end{array}$ & $\hat{\mathrm{a}}$ & $\stackrel{\sim}{n}$ & $\tilde{\infty}$ & $\stackrel{\infty}{+}$ & రి & 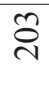 & 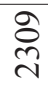 & $\begin{array}{l}\infty \\
\infty \\
n\end{array}$ & $\hat{-}$ & $\stackrel{n}{f}$ & ฮิ & $\begin{array}{l}\infty \\
\infty \\
m\end{array}$ & $\begin{array}{l}0 \\
\stackrel{2}{n}\end{array}$ & Ðે \\
\hline$\stackrel{\Xi}{\circlearrowright}$ & 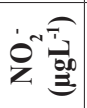 & જे & $\stackrel{\infty}{\sim}$ & $\vec{\sim}$ & $\frac{m}{\gamma}$ & $\stackrel{ \pm}{ \pm}$ & $\stackrel{\infty}{\sim}$ & $\infty$ & $\begin{array}{l}n \\
\infty \\
\infty\end{array}$ & $\frac{2}{n}$ & m & $\frac{0}{m}$ & 亓 & $\stackrel{n}{f}$ & $\bar{I}$ & $\hat{\infty}$ \\
\hline ల్ & 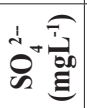 & $\overrightarrow{\mathrm{N}}$ & $\stackrel{0}{\circ}$ & $\stackrel{b}{\oplus}$ & $\vec{\sim}$ & $\begin{array}{l}\infty \\
\stackrel{\sim}{\sim}\end{array}$ & $\stackrel{\circ}{\sim}$ & $\stackrel{\circ}{r}$ & $\stackrel{\sim}{\infty}$ & $\begin{array}{l}\infty \\
\stackrel{\sim}{N}\end{array}$ & $\begin{array}{l}0 \\
\infty \\
\infty\end{array}$ & $\stackrel{\infty}{\sim}$ & $\stackrel{n}{=}$ & $\hat{\infty}$ & 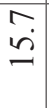 & $\begin{array}{l}0 \\
\infty \\
\infty\end{array}$ \\
\hline है. & 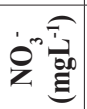 & $\stackrel{m}{+}$ & テr & $\stackrel{m}{\sim}$ & $\stackrel{\nabla}{0}$ & in & $\stackrel{0}{-}$ & $\stackrel{\sim}{\forall}$ & $\stackrel{\nabla}{\forall}$ & $\begin{array}{l}\ddot{1} \\
\ddot{n}\end{array}$ & $\tilde{n}$ & 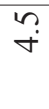 & $\stackrel{N}{m}$ & $\exists$ & $\stackrel{q}{\dot{\nabla}}$ & aे \\
\hline$\frac{\dot{0}}{0}$ & 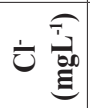 & $\underset{0}{\infty}$ & $\stackrel{?}{\forall}$ & $m$ & 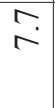 & ?a & $\tilde{n}$ & $\stackrel{0}{\dot{m}}$ & $\stackrel{?}{\sim}$ & $\begin{array}{l}0 \\
\sim\end{array}$ & $\stackrel{\sim}{m}$ & $\stackrel{\Upsilon}{a}$ & $\underset{\infty}{+}$ & ?ִ & $\overrightarrow{0}$ & $\stackrel{\infty}{\circ}$ \\
\hline : & 䒺焉 & $\begin{array}{l}\tilde{n} \\
\tilde{n} \\
\tilde{n}\end{array}$ & $\begin{array}{l}\stackrel{+}{\sim} \\
\infty \\
\infty \\
n\end{array}$ & $\begin{array}{l}\tilde{\sigma} \\
\text { i }\end{array}$ & 尺े. & $\stackrel{\hat{\sigma}}{2}$ & $\underset{\infty}{\stackrel{+}{*}}$ & $\frac{\infty}{n}$ & $\begin{array}{l}\infty \\
0 \\
0\end{array}$ & $\stackrel{m}{\stackrel{m}{\sigma}}$ & $\begin{array}{l}\stackrel{\rho}{\varphi} \\
\infty\end{array}$ & $\frac{0}{m}$ & $\begin{array}{l}\stackrel{g}{q} \\
\gamma\end{array}$ & $\begin{array}{l}I \\
\Xi\end{array}$ & $\begin{array}{l}\circ \\
n \\
n\end{array}$ & $\begin{array}{l}\text { Pे } \\
\text { in }\end{array}$ \\
\hline$\frac{0}{\frac{\pi}{0}}$ & \& & $\begin{array}{l}\text { †े } \\
\stackrel{0}{0}\end{array}$ & 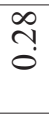 & $\begin{array}{l}0 \\
\stackrel{0}{0}\end{array}$ & 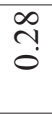 & ֻै & $\begin{array}{l}0 \\
\stackrel{0}{0}\end{array}$ & $\frac{m}{0}$ & $\frac{m}{0}$ & $\frac{n}{0}$ & $\frac{\sim}{\stackrel{0}{0}}$ & ?̊ & 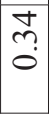 & $\begin{array}{c}0 \\
\stackrel{1}{0}\end{array}$ & 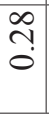 & $\begin{array}{l}\infty \\
\tilde{m} \\
0\end{array}$ \\
\hline$\stackrel{\check{\Xi}}{\varrho}$ & 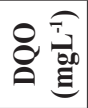 & 므 & in & $\stackrel{\infty}{\sim}$ & ţ & in & $\overparen{\sim}$ & $\underset{+}{\infty}$ & $\tilde{m}$ & n & m & $\stackrel{\infty}{+}$ & $\underset{f}{\mathcal{Y}}$ & ñ & $\stackrel{\infty}{+}$ & $\underset{T}{ \pm}$ \\
\hline 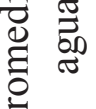 & 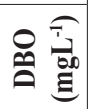 & $\begin{array}{l}\dot{f} \\
\dot{f}\end{array}$ & $\begin{array}{l}\infty \\
\stackrel{N}{N}\end{array}$ & $\hat{a}$ & 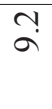 & $\stackrel{\infty}{\infty}$ & 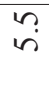 & $\stackrel{\circ}{\circ}$ & $\stackrel{\circ}{\circ}$ & $\underset{\infty}{\infty}$ & $\stackrel{0}{0}$ & $\stackrel{m}{m}$ & $a$ & $\begin{array}{l}0 \\
0\end{array}$ & $\stackrel{\circ}{\circ}$ & $\stackrel{\sim}{\sim}$ \\
\hline & 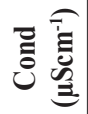 & $\begin{array}{l}8 \\
0 \\
\stackrel{0}{n} \\
m\end{array}$ & $\begin{array}{l}\stackrel{8}{n} \\
\frac{\infty}{\infty}\end{array}$ & $\begin{array}{l}\underset{m}{\infty} \\
\infty \\
\infty\end{array}$ & ?̊ & $\begin{array}{l}n \\
i \\
i \\
i \\
\sim\end{array}$ & $\begin{array}{l}8 \\
8 \\
\text { in }\end{array}$ & $\begin{array}{l}8 \\
\text { in } \\
\text { ñ }\end{array}$ & 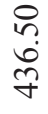 & $\frac{n}{i}$ & $\begin{array}{l}\stackrel{n}{\sim} \\
\stackrel{\text { N }}{n}\end{array}$ & $\stackrel{m}{m}$ & $\begin{array}{l}8 \\
\dot{n} \\
m\end{array}$ & $\begin{array}{l}0 \\
\tilde{n} \\
\\
\text { na }\end{array}$ & 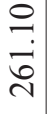 & 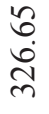 \\
\hline & 音㫕 & $\vec{\partial}$ & $\begin{array}{l}\curvearrowright \\
\text { ஸे }\end{array}$ & $\stackrel{n}{\rightleftharpoons}$ & $\begin{array}{l}\ddot{m} \\
\ddot{n}\end{array}$ & $\frac{n}{6}$ & $\stackrel{n}{6}$ & $\vec{r}$ & $\begin{array}{l}\stackrel{9}{\sim} \\
\dot{\sim}\end{array}$ & $\begin{array}{l}\tilde{b} \\
i\end{array}$ & 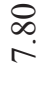 & $\begin{array}{l}\hat{\sigma} \\
9\end{array}$ & $\begin{array}{c}\overline{0} \\
\dot{m}\end{array}$ & ָ̦ & $\begin{array}{l}\text { oे } \\
\text { ì. }\end{array}$ & $\begin{array}{l}\text { So } \\
\text { ¿ें }\end{array}$ \\
\hline & 플 & 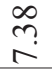 & ભి & సે & $\frac{N}{\pi}$ & $\stackrel{\infty}{\stackrel{\infty}{r}}$ & $\stackrel{\mathbb{N}}{N}$ & テ & $\underset{\stackrel{n}{r}}{r}$ & $\begin{array}{l}\stackrel{8}{0} \\
\end{array}$ & $\hat{\infty}$ & $\stackrel{8}{\circ}$ & $\begin{array}{c}\tilde{N} \\
\infty\end{array}$ & $\bar{\infty}$ & $\underset{\sigma}{\sigma}$ & $\stackrel{n}{n}$ \\
\hline & 它 & $\stackrel{\omega}{m}$ & $\mathscr{2}$ & 当 & $\widetilde{U}$ & 占 & 离 & $\mathbb{\Sigma}$ & $\frac{\mathrm{L}}{\mathrm{S}}$ & $\Leftrightarrow$ & $\overrightarrow{0}$ & 5 & $\Xi$ & $\bar{a}$ & 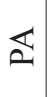 & $\stackrel{\vartheta}{\ominus}$ \\
\hline
\end{tabular}

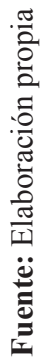


Jorge Herrera-Murillo - Deivis Anchía-Leitón - José Félix Rojas-Marín

Diana Mora-Campos - Alejandra Gamboa-Jiménez - María Chaves Villalobos.

Influencia de los patrones de uso de la tierra en la calidad de las aguas superficiales de la subcuenca del río Virilla, Costa Rica

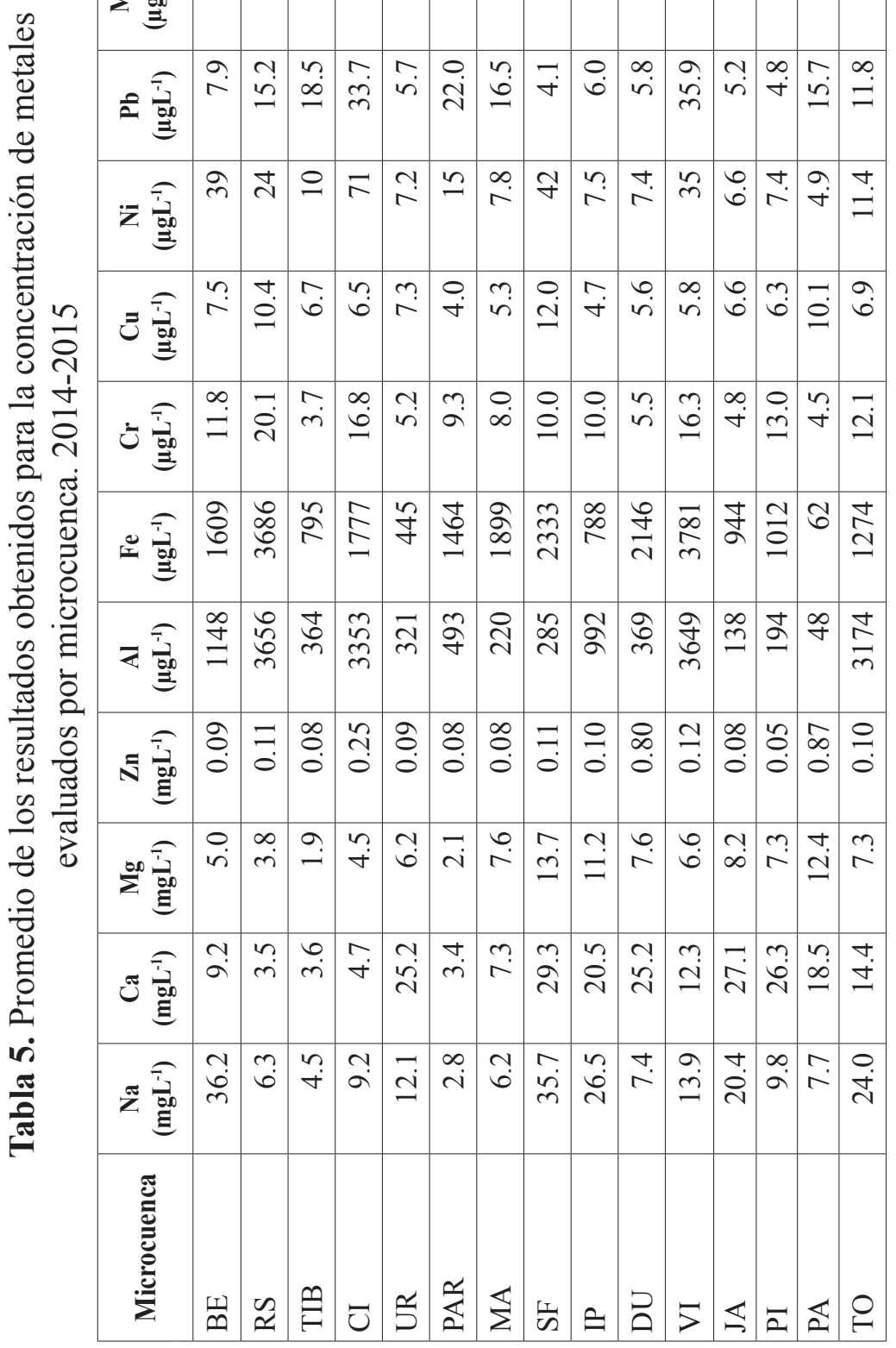

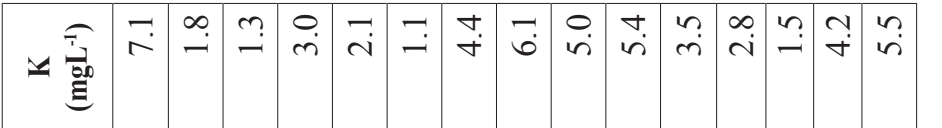

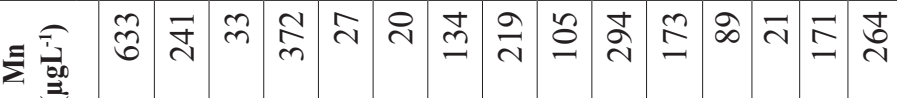

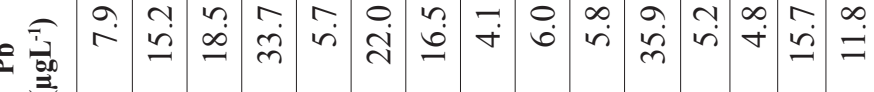

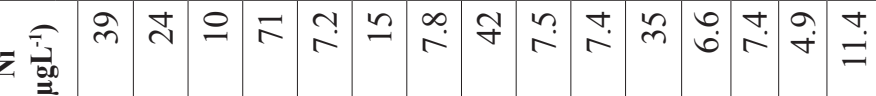

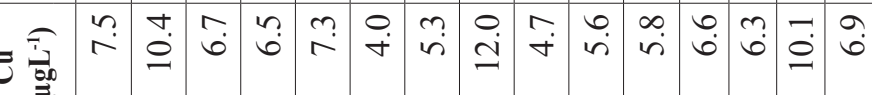

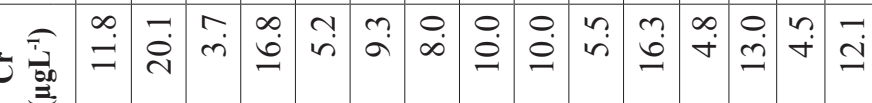

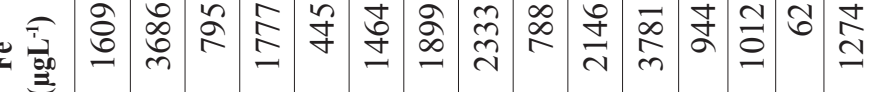

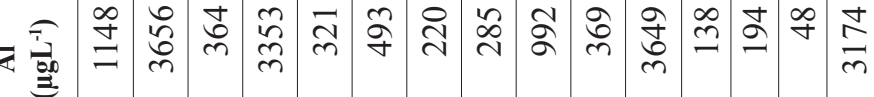

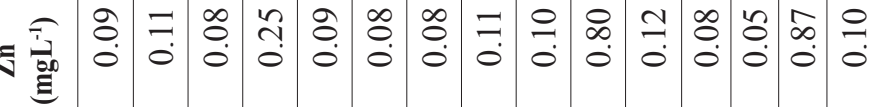

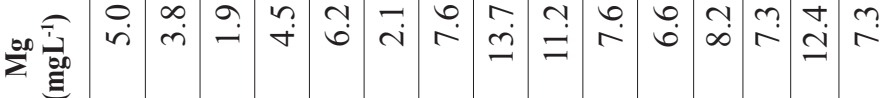

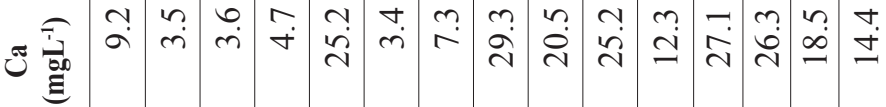

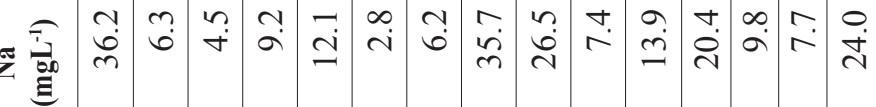

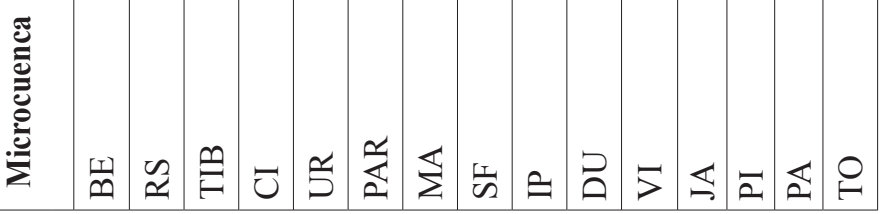

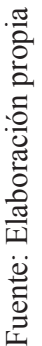


Para la estimación del índice se tomaron en cuenta los siguientes metales: $\mathrm{Al}, \mathrm{Cr}, \mathrm{Cu}, \mathrm{Ni}, \mathrm{Pb}$ y $\mathrm{Mn}$, así como los respectivos valores máximos permitidos, según el tipo de uso, contenidos en el Decreto 33903-MINAES Reglamento para la evaluación y clasificación de la calidad de cuerpos de agua superficiales. Según los valores del índice obtenidos (Tabla 6), todas las microcuencas están seriamente amenazadas con contaminación metálica para el abastecimiento de agua y la preservación de la vida acuática $(\mathrm{MI}>1)$, principalmente en BE, RS, CI y TO. En el caso del riego, TIB, UR, PAR, JA y PI presentan condiciones adecuadas para su uso sin riesgo. Sin embargo, la tasa de adsorción de sodio (TAS), que es una medida de la idoneidad del agua para su uso en el riego agrícola (Myers, 1991), mostró valores entre 0.23 y 2.55 para la totalidad de las microcuencas. La TAS cuantifica la proporción relativa de sodio a iones de calcio y magnesio. En general, cuanto mayor es la relación de adsorción de sodio, menos adecuada es el agua para riego, ya puede requerir enmiendas del suelo para evitar daños a largo plazo (Bahnasawy et al., 2011).

Tabla 6. Índice de metal para las microcuencas que integran la subcuenca del río Virilla, 2015

\begin{tabular}{|c|r|r|r|c|}
\hline \multirow{2}{*}{ Microcuenca } & \multicolumn{3}{|c|}{ Índice de metal } & \multirow{2}{*}{ TAS } \\
\cline { 2 - 4 } & $\begin{array}{c}\text { Abastecimiento } \\
\text { Humano }\end{array}$ & Riego & $\begin{array}{c}\text { Vida } \\
\text { Acuática }\end{array}$ & \\
\hline BE & 20.5 & 4.0 & 25.9 & 2.55 \\
\hline RS & 21.9 & 2.6 & 39.2 & 0.86 \\
\hline TIB & 4.3 & 0.7 & 4.7 & 0.82 \\
\hline CI & 24.8 & 4.0 & 38.3 & 1.00 \\
\hline UR & 2.7 & 0.4 & 3.8 & 0.39 \\
\hline PAR & 5.6 & 0.8 & 6.3 & 0.51 \\
\hline MA & 3.6 & 1.2 & 3.7 & 0.42 \\
\hline SF & 4.6 & 1.7 & 5.1 & 0.83 \\
\hline IP & 6.3 & 1.0 & 11.0 & 0.84 \\
\hline DU & 3.5 & 1.8 & 5.6 & 0.23 \\
\hline VI & 24.3 & 2.8 & 39.6 & 0.74 \\
\hline JA & 1.8 & 0.7 & 2.2 & 0.58 \\
\hline
\end{tabular}


Jorge Herrera-Murillo - Deivis Anchía-Leitón - José Félix Rojas-Marín

Diana Mora-Campos - Alejandra Gamboa-Jiménez - María Chaves Villalobos.

Influencia de los patrones de uso de la tierra en la calidad de las aguas superficiales de la subcuenca del río Virilla, Costa Rica

\begin{tabular}{|c|c|c|c|c|}
\hline \multirow{2}{*}{ Microcuenca } & \multicolumn{3}{|c|}{ Índice de metal } & \multirow{2}{*}{ TAS } \\
\cline { 2 - 4 } & $\begin{array}{c}\text { Abastecimiento } \\
\text { Humano }\end{array}$ & Riego & $\begin{array}{c}\text { Vida } \\
\text { Acuática }\end{array}$ & \\
\hline PI & 2.1 & 0.4 & 2.6 & 0.29 \\
\hline PA & 2.5 & 1.3 & 2.1 & 0.25 \\
\hline TO & 18.4 & 2.4 & 33.9 & 1.11 \\
\hline
\end{tabular}

Fuente: Elaboración propia

La Figura 5 muestra el dendrograma resultante del análisis de clúster basado en los datos de los parámetros fisicoquímicos evaluados en las muestras de agua superficial. A partir del dendrograma, se puede determinar que las 15 microcuencas que integran la subcuenca del río Virilla se agrupan en seis clústers, con el $90 \%$ de la varianza explicada. El primer grupo incluye los ríos $\mathrm{BE}$ y $\mathrm{TO}$ que se caracterizan por poseer un fuerte componente urbano, con aportes secundarios de bosque y agricultura (Tabla 7). En el segundo (UR), tercer (RS) y cuarto grupo (CI, VI, IP) se encuentran microcuencas que se caracterizan por poseer una contribución de similar en proporción para los usos agrícolas, urbano y bosque. El quinto grupo se divide a su vez en dos subgrupos integrados por las microcuencas TIB, PAR, MA, DU y JA, QH, PI caracterizadas por tener una mayor aporte de bosques y pastizales. La diferencia entre ambos subgrupos se encuentran en el hecho de que en el primer caso la predominancia es del uso destinado a pastos sobre bosques, mientras que para el segundo subgrupo se invierte la relación. En la última agrupación se puede encontrar a SF. En este último caso, la separación con respecto al resto de microcuencas se puede deber al hecho de que a pesar de que se presentan tres usos del suelo generalizados, la proporción entre el área urbana con respecto al destino agrícola y bosque es mucho mayor que en las categorías I-IV. 
Figura 5. Dendrograma resultante del análisis de clúster de las 15 microcuencas que integran la subcuenca del río Virilla

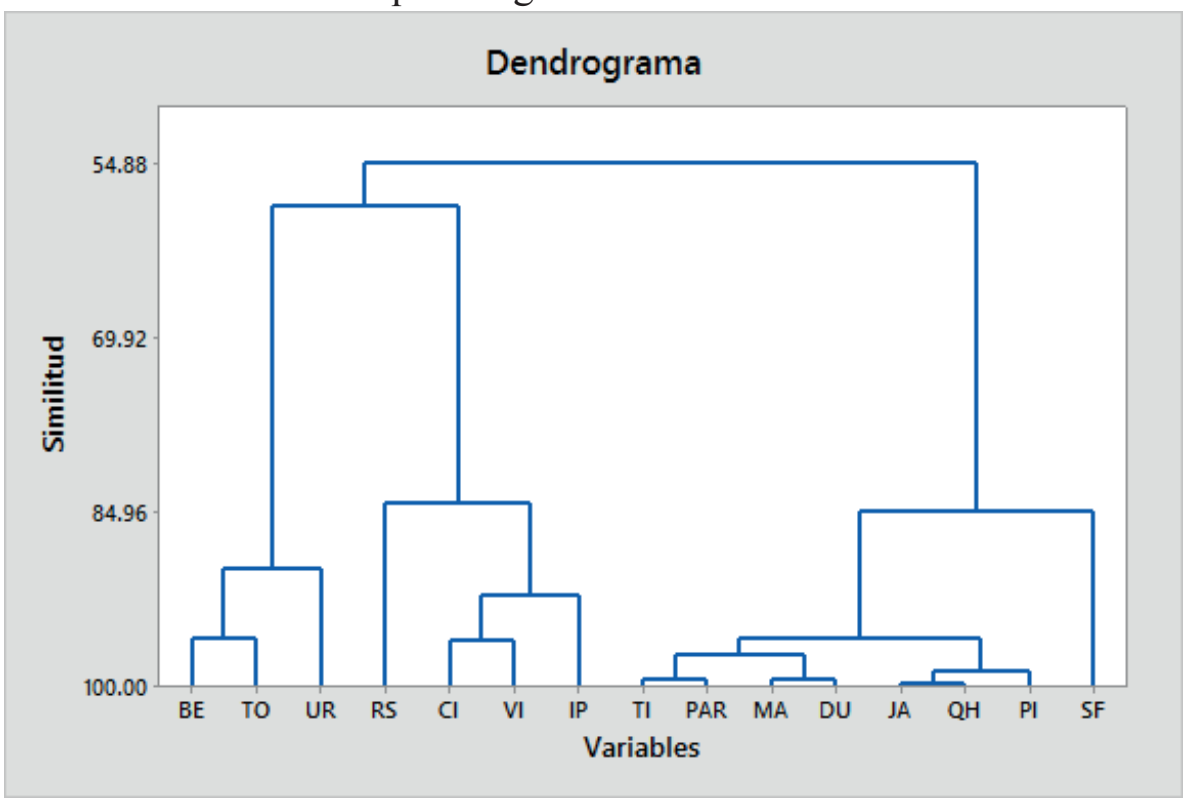

Fuente: Elaboración propia

Tabla 7. Distribución de los porcentajes de área por tipo de uso de la tierra para cada microcuenca. 2015

\begin{tabular}{|c|c|r|r|r|r|r|r|r|}
\hline \multirow{2}{*}{ Código } & \multirow{2}{*}{ Microcuenca } & \multicolumn{7}{|c|}{ Porcentaje de área por tipo de uso de la tierra } \\
\cline { 3 - 9 } & & Agrícola & Urbana & Bosque & $\begin{array}{c}\text { Charral } \\
\text { y Tacotal }\end{array}$ & Pastos & $\begin{array}{c}\text { Terreno } \\
\text { descubierto }\end{array}$ & $\begin{array}{c}\text { Sin } \\
\text { identificar }\end{array}$ \\
\hline BE & Río Bermúdez & 33 & 43 & 14 & 3 & 7 & 0 & 0 \\
\hline RS & Río Segundo & 31 & 19 & 30 & 2 & 16 & 0 & 2 \\
\hline TIB & Río Tibás & 34 & 7 & 21 & 4 & 33 & 0 & 1 \\
\hline CI & Río Ciruelas & 23 & 23 & 21 & 2 & 24 & 0 & 7 \\
\hline UR & Río Uruca & 12 & 5 & 59 & 4 & 18 & 1 & 0 \\
\hline PAR & Río Pará & 1 & 4 & 31 & 2 & 60 & 0 & 2 \\
\hline MA & Río Macho & 0 & 4 & 14 & 3 & 72 & 0 & 7 \\
\hline SF & Quebrada San & 16 & 71 & 12 & 1 & 0 & 0 & 0 \\
\hline Francisco & Río Ipís & 2 & 87 & 4 & 2 & 5 & 0 & 0 \\
\hline
\end{tabular}


Jorge Herrera-Murillo - Deivis Anchía-Leitón - José Félix Rojas-Marín

Diana Mora-Campos - Alejandra Gamboa-Jiménez - María Chaves Villalobos.

Influencia de los patrones de uso de la tierra en la calidad de las aguas superficiales de la subcuenca del río Virilla, Costa Rica

\begin{tabular}{|c|c|c|c|c|c|c|c|c|}
\hline \multirow{2}{*}{ Código } & \multirow{2}{*}{ Microcuenca } & \multicolumn{6}{|c|}{ Porcentaje de área por tipo de uso de la tierra } \\
\cline { 3 - 9 } & & Agrícola & Urbana & Bosque & $\begin{array}{c}\text { Charral } \\
\text { y Tacotal }\end{array}$ & Pastos & $\begin{array}{c}\text { Terreno } \\
\text { descubierto }\end{array}$ & $\begin{array}{c}\text { Sin } \\
\text { identificar }\end{array}$ \\
\hline DU & Río Durazno & 0 & 3 & 36 & 4 & 54 & 0 & 2 \\
\hline VI & Río Virilla & 15 & 26 & 28 & 5 & 18 & 0 & 9 \\
\hline JA & Río Jaris & 1 & 0 & 79 & 0 & 20 & 0 & 0 \\
\hline PI & Río Picagres & 11 & 4 & 52 & 5 & 23 & 3 & 2 \\
\hline PA & Río Pacacua & 9 & 6 & 50 & 4 & 30 & 0 & 0 \\
\hline TO & Río Torres & 12 & 38 & 31 & 6 & 12 & 0 & 1 \\
\hline
\end{tabular}

Fuente: Elaboración propia

La agrupación obtenida del análisis clúster permite una mayor comprensión del comportamiento de los parámetros fisicoquímicos dentro de la subcuenca del río Virilla. Así por ejemplo, los valores de DBO, DQO y SST tiende a ser menores conforme se avanza en las categorías, indicando que los grupos I, II, III y IV tienen un mayor aporte antropogénico proveniente de la descarga de aguas residuales de origen urbano. Los resultados obtenidos en el análisis de clúster coinciden con los datos derivados de la matriz de correlación de Spearman, la cual se aplicó dada la distribución no normal de los datos. En la Tabla 8, se muestra que para los grupos I y II se registra una correlación positiva con el pH, DBO, DQO, SST y los metales $\mathrm{Cr}, \mathrm{Cu}, \mathrm{Pb}$ y $\mathrm{Zn}$. Sin embargo, en estos grupos, donde predomina el uso urbano de la tierra, se correlacionó negativamente la turbiedad y DQO. Adicionalmente, se observó una relación no significativa del uso del suelo urbano con la temperatura, la conductividad, amonio, nitrato, fosfato, entre otros. En el caso de los grupos III y IV donde se presenta un uso de la tierra mixto (urbano-agrícola-bosque), se obtuvo un patrón similar a los grupos I y II en el caso del DBO, DQO y SST con la incorporación de una correlación positiva significativa con turbidez-nitrato, Ptotal-nitrato, magnesio-nitrato y correlación negativa de Ca y Mg con la DQO. Sin embargo, la correlación con otros parámetros, a saber, temperatura, conductividad, $\mathrm{pH}, \mathrm{DBO}$ y fosfato, no fue significativa. $\mathrm{El} \mathrm{Mg}$ y el $\mathrm{NO}_{3}{ }^{-}$son nutrientes provienen principalmente de la agricultura (tierras cultivables y pastizales) (Bahar et al., 2008). 
Tabla 8. Patrones de correlación de Spearman significativos de acuerdo con la clasificación de microcuencas obtenidas por análisis de clúster.

\begin{tabular}{|c|c|c|c|}
\hline \multicolumn{4}{|c|}{ Grupos derivados del análisis de clúster } \\
\hline I y II & III y IV & $\mathbf{V}$ & VI \\
\hline $\begin{array}{l}\text { DBO vrs DQO } \\
(0.825)\end{array}$ & $\begin{array}{l}\text { DBO vrs DQO } \\
(0.748)\end{array}$ & $\begin{array}{l}\text { Na vrs K } \\
(0.885)\end{array}$ & $\begin{array}{l}\text { DBO vrs DQO } \\
(0.781)\end{array}$ \\
\hline $\begin{array}{l}\text { DBO vrs SST } \\
(0.827)\end{array}$ & $\begin{array}{l}\text { DBO vrs SST } \\
(0.703)\end{array}$ & $\begin{array}{l}\text { Na vrs Turbiedad } \\
(0.917)\end{array}$ & $\begin{array}{l}\text { DBO vrs SST } \\
(0.767)\end{array}$ \\
\hline $\begin{array}{l}\text { DQO vrs SST } \\
(0.842)\end{array}$ & $\begin{array}{l}\text { DQO vrs SST } \\
(0.815)\end{array}$ & $\begin{array}{l}\text { K vrs Turbiedad } \\
(0.832)\end{array}$ & $\begin{array}{l}\text { DQO vrs SST } \\
(0.840)\end{array}$ \\
\hline $\begin{array}{l}\text { pH vrs DQO } \\
(0.677)\end{array}$ & $\begin{array}{l}\text { Turbiedad vrs NO3- } \\
(0.792)\end{array}$ & $\begin{array}{l}\text { Turbiedad vrs Cl- } \\
(0.753)\end{array}$ & $\begin{array}{l}\text { Turbiedad vrs Cl- } \\
(0.693)\end{array}$ \\
\hline $\begin{array}{l}\mathrm{Cr} \text { vrs DQO } \\
(0.651)\end{array}$ & $\begin{array}{l}\text { P total vrs NO3- } \\
(0.648)\end{array}$ & $\begin{array}{l}\text { Ca vrs } \mathrm{Mg} \\
(0.719)\end{array}$ & $\begin{array}{l}\text { Turbiedad vrs NO3- } \\
(0.715)\end{array}$ \\
\hline $\begin{array}{l}\mathrm{Cu} \text { vrs DQO } \\
(0.572)\end{array}$ & $\begin{array}{l}\text { Mg vrs NO3- } \\
(0.871)\end{array}$ & $\begin{array}{l}\text { Ca vrs SST } \\
(0.839)\end{array}$ & $\begin{array}{l}\text { Zn vrs SST } \\
(0.725)\end{array}$ \\
\hline $\begin{array}{l}\mathrm{Pb} \text { vrs SST } \\
(0.625)\end{array}$ & $\begin{array}{l}\text { Ca vrs DQO } \\
(-0.594)\end{array}$ & $\begin{array}{l}\text { Zn vrs SST } \\
(0.793)\end{array}$ & \\
\hline $\begin{array}{l}\text { Zn vrs SST } \\
(0.688)\end{array}$ & $\begin{array}{l}\text { Mg vrs DQO } \\
(-0.547)\end{array}$ & $\begin{array}{l}\text { Conductividad vrs } \mathrm{Na} \\
(0.718)\end{array}$ & \\
\hline $\begin{array}{l}\text { Turbiedad vrs DQO } \\
(-0.706)\end{array}$ & & $\begin{array}{l}\text { Turbiedad vrs NO3- } \\
(0.747) \\
\text { P total vrs NO3- } \\
(0.711)\end{array}$ & \\
\hline
\end{tabular}

Fuente: Elaboración propia

Para el grupo V, el patrón de correlaciones presentó diferencias importantes con respecto a los anteriores, relacionando parámetros como Na$\mathrm{K}$, Na-turbiedad, K-turbiedad, $\mathrm{Ca}-\mathrm{Mg}$, turbiedad-cloruro, $\mathrm{Ca}-\mathrm{Mg}$, entre otros, los cuales evidencian el aporte de la litología de los cuerpos de agua, 
así como el aporte de fuentes difusas relacionadas con procesos de erosión (Miller et al., 2011). En el último grupo (VI) se evidenciaron correlaciones entre DBO, DQO, SST, turbiedad, cloruro y nitrato.

Con el fin de identificar las principales fuentes de contaminación, se realizó un análisis de factor sobre los datos normalizados obtenidos para los grupos mediante el análisis de clúster. El análisis de factor es un método estadístico multivariado que se puede utilizar para describir la variabilidad entre los parámetros observados en términos de un menor número de mensurandos no observados llamados factores (Tabachnick y Fidell, 2001). Los resultados del análisis de factor junto con los tipos de fuentes probables se presentan en la Tabla 9. Según Liu et al. (2003), cargas factoriales $>0.75,(0.5-0.75)$ y $(0.3-0.5)$ se consideran como fuertes, moderadas y débiles, respectivamente. Para los grupos 1 y 2 , se obtuvieron tres varifactores que explican el $84.3 \%$ de la varianza total. El primer varifactor (VF1), representa el $18.1 \%$ de la varianza total y presenta una fuerte correlación entre turbiedad, cloruro, $\mathrm{Na}, \mathrm{K}$ y Ca que relaciona los factores naturales como la litología y los tipos de suelo. El segundo varifactor (VF2), definió el $52.5 \%$ de la varianza total e incluyó la pH, DQO, DBO y SST. Este factor representa múltiples fuentes de contaminación y puede ser generado, principalmente por la escorrentía urbana y aguas residuales industriales. Por su parte el VF3 relaciona el Cl$, \mathrm{SO}_{4}^{2-}, \mathrm{NO}_{3}^{-}, \mathrm{NH}_{4}^{+}$, $\mathrm{Ca}$ y $\mathrm{Mg}$ puede provenir de las actividades agrícolas que se desarrollan en las microcuencas. En el caso de los grupos 3 y 4, se generan factores que explican la variabilidad de los datos semejantes al primer caso, sin embargo, el porcentaje de contribución de cada uno presenta variaciones con respecto al grupo 1 y 2 , sobre todo, debido a la reducción del aporte urbano. Para las microcuencas localizadas en el grupo 5, la situación resulta totalmente diferente, ya que la variabilidad de los datos se explica por dos factores mayoritariamente, factores litológicos y actividades agrícolas, representando el $80.5 \%$. 
Jorge Herrera-Murillo - Deivis Anchí-Leitón - José Félix Rojas-Marín

Diana Mora-Campos - Alejandra Gamboa-Jiménez - María Chaves Villalobos.

Influence of Land Use Patterns in the Quality of Surface Waters of the Virilla River Sub-Basin, Costa Rica

on

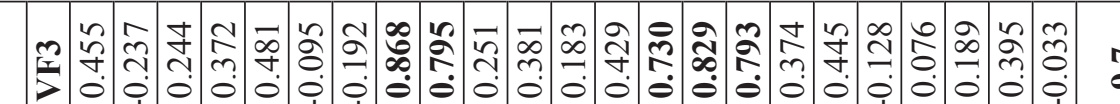

ڤ

苞

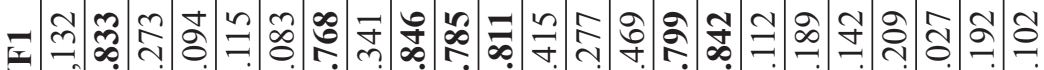

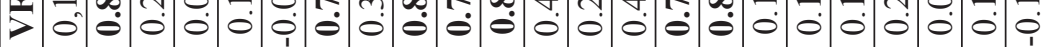

$\hat{\circ}$

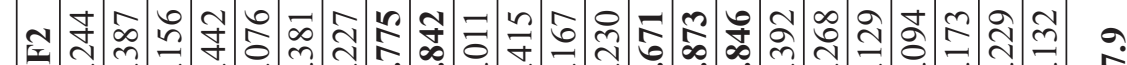

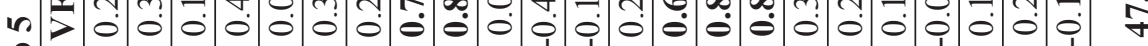

䒠

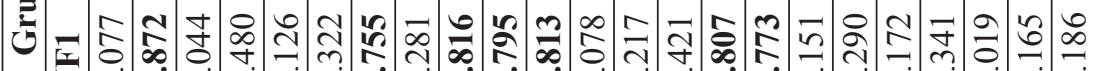

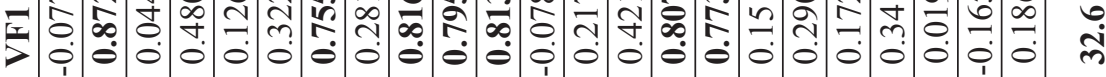

으 금

를

is $\cdot \Omega$

䨌

สี

光

?

$+$

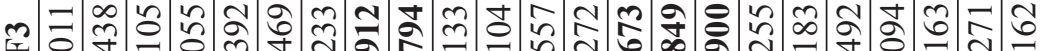

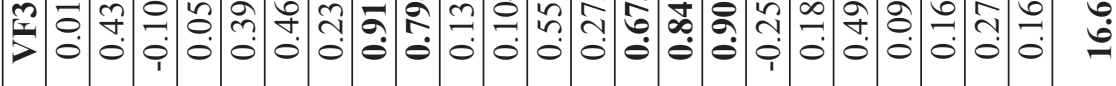

m

岁

(5)

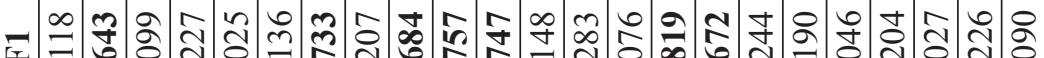

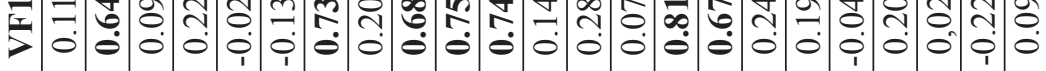

$\stackrel{2}{\frac{1}{2}}$

m

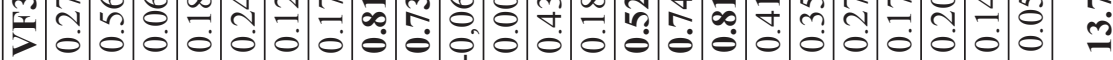

4

พ

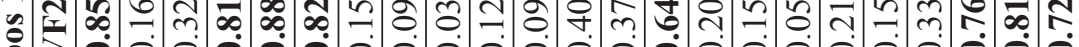

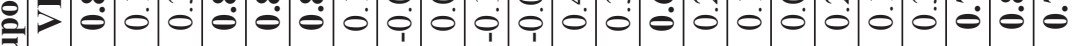

กิ

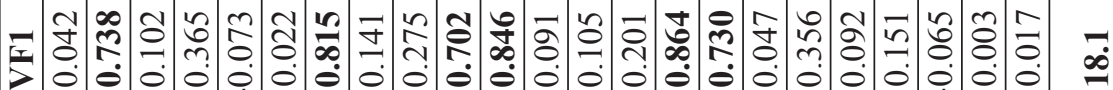

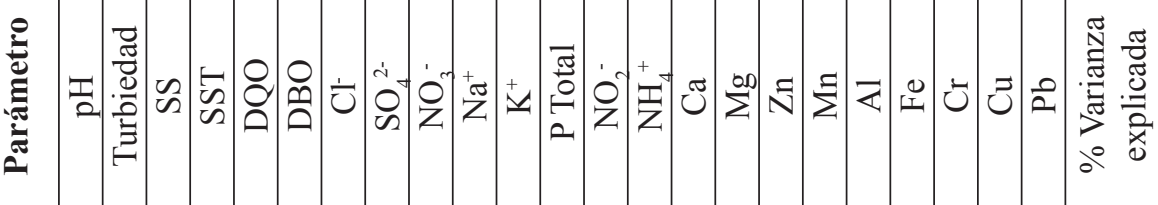


Para la microcuenca SF, incluida en el grupo 6, el análisis de factor dio como resultado la presencia de un factor VF2 que explica el $60.4 \%$ de la variabilidad y que contiene parámetros como el pH, DBO, DQO y SST, que como se mencionó anteriormente está asociado a la descarga de aguas residuales sin tratamiento.

\section{Conclusiones}

A partir de los resultados obtenidos en la presente investigación, se puede concluir que las microcuencas que componen la subcuenca del río Virilla pueden ser clasificadas en seis categorías según el análisis de conglomerados. Estas categorías corresponden directamente con las diferencias en los patrones de uso de la tierra que predominan en cada una de ellas, de forma tal que las áreas que poseen importantes entornos urbanos se rigen por el aporte de la descarga de aguas residuales sin tratamiento, lo que se refleja en la presencia de altas concentraciones de DBO, DQO y SST en los cuerpos de agua superficial. Por otra parte, las microcuencas con predominio de bosques y pastos, presentan una variabilidad química en la calidad de sus aguas que puede ser explicada mayoritariamente por el aporte litológico y la escorrentía de sedimentos provenientes de zonas agrícolas y pastizales.

Dado que la dependencia de los impactos del uso del suelo en la escala espacial es diferente para los distintos contaminantes, es importante identificar y evaluar el aporte de las diversas fuentes de variabilidad como insumo para ser considerado en la generación de políticas que promuevan una gestión y conservación integral de la tierra, implementada en toda la subcuenca para abordar las múltiples preocupaciones relacionadas con la calidad del agua.

\section{Referencias}

Bahar, M., Ohmori, H. y Yamamuro, M. (2008) Relationship between river water quality and land use in a small river basin running through the urbanizing area of Central Japan. Limnology, 9 (1), 19-26.

Bahnasawy, M., Khidr, A., Dheina, N. (2011) Assessment of heavy metal concentrations in water, plankton and fish of Lake Manzala, Egypt. Turk. J. Zool., 35 (2), 271-280 
Carbone, M. E., García, B., Piccolo, M. C. \& Perillo, G. M. (2013). Impacto Antrópico en la Calidad del Agua Superficial de la Cuenca Media del Arroyo Claromeco. Cuadernos de Investigación Geográfica, 556 $-563$.

Centro de investigaciones Agronómicas (CIA) (2017). Mapa Digital de Suelos en Costa Rica. Recuperado de http://www.cia.ucr.ac.cr/ $10 / 03 / 2017$

Cisneros, R. C. (2005). Uso del suelo y calidad del agua en quebradas de fincas con sistemas silvopastoriles en la Subcuenca del Río Jabonal, Costa Rica. Turrialba, Turrialba, Costa Rica.

Kaushal, S. \& Belt, K. T. (2012) The urban watershed continuum: evolving spatial and temporal dimensions. Urban Ecosyst.15, 409-435.

Instituto Meteorológico Nacional (IMN) (2017). Régimen de precipitación en Costa Rica. a. Recuperado de https://www.imn.ac.cr/web/imn/ inicio 13/04/2017

Instituto Nacional de Estadísticas y Censos (INEC) (2011). Censo Nacional de Población 2011. Recuperado de https://www.inec.go.cr/ 08/06/2017

Liu, C.W., Lin, K.H. \& Kuo, Y.M. (2003). Application of factor analysis in the assessment of groundwater quality in a Blackfoot disease area in Taiwan. Science in the Total Environment 313(1-3), 77-89.

Ministerio de Agricultura y Ganadería (MAG) (2007). Reforma Metodología Determinación Capacidad Uso Tierras osta Rica, Decreto $\mathrm{N}^{\circ}$ 23214-MAG-MIRENEM. Procuraduría General de la República de costa Rica. Recuperado de http://www.pgrweb.go.cr/scij/Busqueda/Normativa/Normas/nrm_texto_completo.aspx?param $1=\mathrm{NR}$ TC\&nValor $1=1 \&$ nValor $2=60982 \&$ nValor $3=69013 \&$ strTipM $=$ TC $05 / 06 / 2017$

Miller, J. D., Schoonover, J.E., Williard, K. \& Hwang, C. (2011) Whole catchment land cover effects on water quality in the Lower Kaskaskia River Watershed. Water Air Soil Pollut, 221, 337-350.

Miserendino, M. L., Casaux, R., Archangelsky, M., Di Prinzio, C. Y., Brand, C. \& Kutschker, A. M. (2011). Assessing land-use effects on water quality, in-stream habitat, riparian ecosystems and biodiversity in Patagonian northwest streams. Sci. Total Environ. 409(3), 612-624. 
Quesada, M. (2012). Dinámica territorial en el uso del suelo y el régimen hidrológico: región central, Costa Rica. Espacio y Desarrollo, 45-56. Sangani MH, Amiri BJ, Shabani AA, Sakieh Y. \& Ashrafi S (2015) Modeling relationships between catchment attributes and river water quality in southern catchments of the Caspian Sea. Environ Sci Pollut Res. 22, 4985-5002

Saroj, G., Techato, K., Monprapussorn, S. \& Yuangyai, C. (2013). Integrating Land use and Water quality for Enviromental based land use planning for U-Tapao River Basin, Thailand. Procedia - Social and Behavioral Sciences. Sciencedirect (91), 556 - 563.

Songyan, Y., Zongxue, X., Wei, W. \& Depeng, Z. (2016). Effect of land use types on stream water quality under seasonal variation and topographic characteristics in the Wei River basin, China. Ecol. Ind. 60, 202-2012.

Tabachnick, B. G. \& Fidell, L. S. (2001). Using Multivariate Statistics. Boston. MA: Allyn and Bacon.

Tamasi, G. \& Cini, R. (2004). Heavy metals in drinking waters from Mount Amiata. Possible risks from arsenic for public health in the province of Siena. Sci. Total Environ. 327, 41-51

Tong, S. T., Liu, A. J. \& Goodrich, J. A. (2009). Assessing the water quality impacts of future land-use changes in an urbanising watershed. Civil Engineering and Environmental Systems, 26(1), 3-18.

Uriarte M, Yackulic CB, Lim, Y. \& Arce-Nazario JA (2011) Influence of land use on water quality in a tropical landscape: a multi-scale analysis. Landsc Ecol 26,1151-1164

Walling, D. E. \& Fang, D. (2003). Recent trends in the suspended sediment loads of the world's rivers. Global and Planetary Change, 39(1-2), 111-126.

Wear, D. N., Turner, M. G. \& Naiman, R. J. (1998). Land cover along an urban-rural gradient: implications for water quality. Ecological Applications, 8(3), 619-630. 